\title{
The management of musculoskeletal pain and disfunction from traffic accidents polytrauma
}

Monica Adriana Farago, Manuela Simona Pop

University of Oradea, Faculty of Medicine, The Municipal Hospital of Oradea, Romania

Peer-reviewed by: Liviu Lazăr, University of Oradea, Faculty of Medicine and Pharmacy

Florina Ojoga, National Institute of Rehabilitation, Physical Medicine and Balneology, “Carol Davila” University of Medicine and Pharmacy

\section{Abstract}

After complex orthopaedic-surgical treatment of car crash polytrauma, we face multilevel patient problems, from algodisfunctional to psychological consequences, which are very difficult to manage. Aim of the study: identifying the optimal ways to approach cases of polytrauma with major functional and psychological implications. Material and Methods: We present the cases of two female patients, 19 and 18-year-old, victims of major road accidents that required car extrication. The accidents resulted in minor craniocerebral trauma, weight bearing bones and pelvis fractures, musculoskeletal and skin avulsion, and neurogenic bladder with catheterization. They underwent repeated orthopedic-surgical interventions and plastic surgery after which they were referred to functional rehabilitation. In the rehabilitation department we were confronted with anxious, uncooperative patients, with a very high level of somatic pain, incapable of actively mobilizing in the bed plan, one of them, within the limits of physical resources, presenting agitation and night aggressiveness. Results: After the complex clinical evaluation we initiated the rehabilitation treatment. The initial rehabilitation results were minimal because of the pain threshold, high levels of anxiety and fatigue due to insomnia. We asked for psychological evaluation and counseling and we organized 6 short daily exercise sessions, then four longer ones, so as not to exceed the patients' momentary resources. The level of difficulty of the kinetic training was progressively increased, being constantly adapted to the daily training gains of the patients. The aggressive outbursts in one of the patients were related to the dread of darkness and loneliness at night, as a consequence of incarceration from the night road accident. In 4 weeks we gained independence for eating, spontaneous urination, bedside independent mobilization, verticalisation and walking with a frame for short distances. Conclusions: Pain, somatic dysfunction and posttraumatic stress management is a complex process that requires a multidisciplinary approach, including the psychological one. The rehabilitation particularity consisted of multiple daytime rehabilitation sessions adapted to the daily resources of the patients.

Key words: car crash, pain management, rehabilitation

Introduction. Car crashes continue to occur. Young and beginner drivers are most often involved in car accidents, frequently followed by death or functional and psychological disabilities. Society has to be prepared not only to prevent road traffic injuries, but also to mitigate their consequences and enhance the quality of life for people who are injured. This involves consistent and well developed education, medical and social resources.

The chances and the quality of survival depend on three components of post-crash care: prehospital, hospital and rehabilitation [1]. Rehabilitation is the intermediate station between acute or emergency care and home, family or institutional environment $[1,2]$. Based on the concept of "Comprehensive inpatient rehabilitation", the case management team addresses specific medical and rehabilitation needs, particular to each case [1].

Material and Methods. We present the cases of two female patients, 19 and 18 years old, victims of road accidents with polytrauma. They both suffered minor traumatic brain injury and multiple fractures

and soft tissue injuries (table1, fig.1,2), which needed intensive care unit assistance, repeated orthopedic and plastic surgery performed on priorities and risks; no organs, spinal cord or large vessels severe lesions; catheterization for neurogenic urine bladder in both cases. They were referred to the rehabilitation department at 6 weeks (for study case 1) and 3 weeks (for study case 2) after the traumatic event.

Table 1. Orthopaedic lesions of the study cases.

\section{Table 1. Orthopedic lesions from the study cases}

Case 1 (EK, 19 years)

Case 2 (OA, 18 years)

- Anterior left rib arch fracture

- Compound pelvic fracture

operated on the right acetabulum

- Femoral shaft fracture - operated

- 2nd, 3rd, 4th metatarsal fracture operated

- Right calf contusion with lack of soft tissue material - plastic surgery treatment

- Incomplete traumatic sciatic nerve injury.
- Nondisplaced both side pelvic fracture - Distal left femoral shaft fracture surgically treated - $3 r d, 4$ th, 5th under capital left metatarsal fractures

- Left medial malleoli nondisplaced fracture 


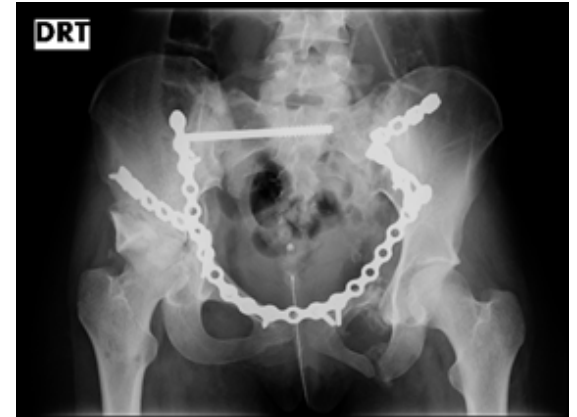

Figure1. Study case 1 (E.K. ,19 yrs ). Right sacroiliac joint instability fixed with transversal screw. Left sacroiliac joint instability fixe by plate. Right ileum, acetabulum and iliopubic bone fixed by modulated plate. Left acetabulum and anterior acetabular wedge fixed by modulated plate. Left ischiopubic bone spontaneous healing.
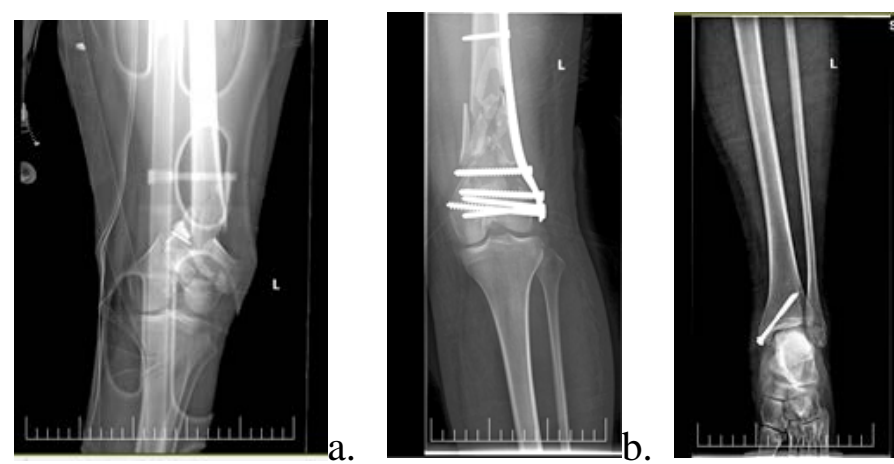

C.

Figure 2. Study case 2 (O.A., 18 yrs).a). Comminutive supracondylar and intercondylar fracture (bone extrusion) left femur; b).Reduction under image intensifier and fixation with slided plate; c). Nondisplaced left medial malleolus fracture fixed with two cancellous screws under image intensifier.

\section{Red flag for the rehabilitation team:}

F Patients' age particularities: young adults with formal education in curse, without work history and living resources, with minimal life experience and totally dependent on family complex care. It is obvious that the consequences of polytrauma might mark the whole evolution of the patients' life, as well as their families' life.

Case management plan: subjective complaints, medical history details, physical exam, laboratory tests, image exams if necessary, the structure of interdisciplinary team, short term, medium term and long term rehabilitation goals, case management team.
Results. Subjective complaints from the study cases: high levels of pain (particularly in study case 1), severe body disfunction, insomnia with nightmares when falling asleep.

Yellow flag for rehabilitation team:

The psycho-affective status of the patients - marked anxiety, severe difficulty in communication, aggressiveness (particularly night physical aggressiveness in study case 1 and verbal aggressiveness in both cases), collaboration difficulties. Creates the premises of the multidisciplinary team intervention need.

Table 2. The physical examination of the cases details

\begin{tabular}{|l|}
\hline Table 2. Objective in Stud \\
\hline \multicolumn{2}{|c|}{ Study case 1 } \\
\hline - Extendense \\
spontaneous pain and muscle \\
masses palpation pain, \\
- Position in bed: right half \\
side lying with right hip and \\
knee flexum, right ankle \\
extension, right foot dorsal \\
flexion being impossibile. \\
(This position is generating \\
muscle imbalances at other \\
levels too.) passive mobility \\
- Severe prer other \\
limitations to the \\
uninvolved joints, not \\
necessarily correlated with \\
pain. \\
- The impossibility of active \\
mobilization in the bed \\
(subjective, because of pain). \\
- Assistance needed for \\
feeding, drinking and general \\
care.
\end{tabular}

\begin{tabular}{|l|l|}
\hline \multicolumn{2}{|c|}{ Mental } \\
\hline - Marked anxiety. & - Marked anxiety. \\
- Low communication & - Low communication capacity \\
capacity because of: pain, & because of: pain, anxiety, lack of \\
anxiety, lack of motivation, & motivation, others? \\
others? & - Low attention span (in the first \\
- Verbal aggressiveness with & two rehabilitation weeks). \\
entourage (within the limit of & - Verbal aggressiveness with the \\
exhaustion) (very limited & mother. \\
physical resources). & - Crying easily. \\
- Nightly verbal and motor & \\
aggressiveness (within the \\
limit of exhaustion) (very \\
limited physical resources). \\
- Amnesia (at admission in \\
rehabilitation department) \\
connected with the accident; \\
memories of the traumatic \\
event regained during \\
hospitalization. \\
\hline \multicolumn{2}{|c|}{ Physical and psychological resistance to interventional } \\
\hline
\end{tabular}


Noticing the psychological status of the patients we considered the need of a psychological evaluation and support.

Psychological assessment
Case 1. Patient with perceptual sensory capacity in
normal relations. She presents an adaptation
anxiety-depressive disorder after the physical
trauma (road accident) with post-operative
temporal functional disability. Irritability,
pessimistic disposition, negative attitude,
dysfunctional affective reactions.
Case 2. Patient mentally developed and with social
maturity according to chronological age. She
presents posttraumatic status disorder (road
accident): anxiety-depressing states, easy crying,
stressful recurrent and intense event memories,
intense psychological distress when exposed to
stimuli, startle response to certain stimuli.

In conclusion, we were confronted with:

- high level of pain and disfunction,

- psycho-affective and emotional imbalance,

- physical and mental fatigue, whose correction became treatment objectives, the basic methodology being pharmacological therapy, exercise therapy (massage being contraindicated, patients being on anticoagulant drugs), psychological counseling.

\section{Pain management in study cases}

$\rightarrow$ Pain is a multidimensional phenomenon commonly associated with trauma or illness and involves pain and suffering experiences and which is usually associated with incapacity and emotional distress $[3,4]$.

$\rightarrow$ Effective pain management in our patients consisted of:

o identifying the multiple medical, psychological, interpersonal and spiritual factors that compete in maintaining the pain and their management;

o preparing an individual approach plan;

0 reassessments of the effectiveness of pain management measures;

o educating the accompanying mother (who was allowed to take part in the patient care).

$\rightarrow$ Identified sources of pain: post-traumatic lesions in evolution, pain from joint stiffness and muscle contractures, somatic pain from stress muscle contraction, but possible psychogenic pain was also taken into account.

$\rightarrow$ Obviously, pain control medication would not have been enough.

Physical disfunction management

$\rightarrow$ Pain control

$\rightarrow$ Physical relaxation technique (applied by physiotherapist)

$\rightarrow$ Corrected postures training

$\rightarrow$ Training joint range of motion and flexibility

$\rightarrow$ Muscle strength and endurance training (for active mobilisation to become possible)

$\rightarrow$ Active mobilisation training:

o In the bed plan - initialy, followed by:

o Transfer from lying position to sitting position (in the plan of the bed and to the bedside)

o Transfer from siting to standig (standing with aids: walker for study case 1 and walker then canes for study case 2)

$\rightarrow$ Balance and coordination training

$\rightarrow$ Gate training (with walker for study case 1 and crutches for study case 2).

$\rightarrow$ Obviously isolated kinetic training would not have been enough.

\section{Neurogenic urine bladder management:}

$\rightarrow$ Urethral catheter was present in both patients

$\rightarrow$ We searched for signs of bladder infection and hematuria

$\rightarrow$ Pelvic floor exercises to prepare the urinary continence (very difficult in study case 1 because of the multiple pelvic girdle operated fractures)

$\rightarrow$ Repeated attempts to remove the urethral catheters to check for bladder control. Finally, the bladder control was achieved.

\section{Mental disorder management:}

$\rightarrow$ On the premise that in the posttraumatic rehabilitation process the mind cannot be separated from the body, we have requested psychological counseling throughout the hospitalization of the patients [5].

$\rightarrow$ Obviously, isolated psychological counseling would not have been enough. 


\section{Rehabilitation team:}

To achieve the treatment goals, we worked in a complex rehabilitation team consisting of: case manager - the rehabilitation physician, physiotherapist, psychologist, medical nurse, cleaning staff and the mothers (who were taught about the needs of the two girls, how to manage them and how to continue with the exercises after discharge, to continue to improve the physical fitness at home). We can say that we have come close to the concept of "COMPREHENSIVE INTEGRATED INPATIENT REHABILITATION"[1, 2].

How did we choose to work to have a favorable evolution of the cases? Our option was:

$\rightarrow$ Continuing the indicated drug treatment from surgical services.

$\rightarrow$ Pain control medication.

$\rightarrow$ Initiation of rehabilitation treatment in bed (to prevent the patients' exhaustion due to the transport to the gym)

$\rightarrow$ Relaxation techniques (very important for both physical and mental relaxation and pain control)

$\rightarrow$ Short physical therapy sessions, repeated throughout the day to cover more areas of interest and to prevent the risk of more pain, muscle fatigue and patient exhaustion, Initially there were 6 sessions / day then, when the patients' tolerance to effort increased, 4 sessions / day, lasting increasingly longer.

$\rightarrow$ Mother's participation (accompanying person) in the rehabilitation treatment, including training for "activities of daily living", and to repeat the exercise throughout the day, independent of the rehabilitation sessions; we need to mention that it was not possible in the very first rehabilitation week, due to the patients psychological negative reaction.

$\rightarrow$ Daily psychological counseling.

\section{Patients rehabilitation achievements:}

Under the conditions of orthopedic lesions and their conservative and postoperative evolution we were able to obtain for this rehabilitation stage:

- Decrease of pain, but not complete disappearance in both cases

- Easy mobilization in the bed

- Transfer at the edge of the bed

- Getting out of the bed

- Walking with frame in study case 1 and with Canadian crutches in study case 2
- Self-feeding

- Toilet hygiene

- Satisfactory momentary psychological balance with sleep improvement; reduction of verbal and motor aggressiveness in study case 1 ; study case 2 managed, during hospitalization, to complete the term test papers in order to be able to participate at the baccalaureate.

\section{Incidents during hospitalization in the rehabilitation department:}

$\rightarrow$ Catheter-associated urinary tract infection in both cases

$\rightarrow$ Study case 1 :

o Right heel stage III bedsore

o Acute gastritis, acute cholecystitis (due to high level of stress and food mistakes - the benevolent family brought her junk food). The patient was 6 weeks after the accident, with a period of inability to eat and then with very limited nutrition.

o According to the mother's statements, her daughter visibly lost weight. At admission, we would have appreciated her as being severely underweight.

\section{The prognostic of the study cases: \\ $>$ Study case 1}

0 With adequate rehabilitation and psychological assistance, long-term case evolution might be good, but being from the countryside, at a great distance from a rehabilitation center, the easy access to these services is lacking, so we have reserves regarding a good functional rehabilitation. We rely on what her mother has learned and is able to apply and on another admission for new rehabilitation steps as well.

0 In addition, because of the right acetabulum fracture, we can expect her to develop aseptic osteonecrosis of the femoral head with all its consequences.

o Osteosynthesis of the pelvic girdle raises reserves about carrying a pregnancy.

\section{$>$ Study case 2}

o The traumatic lesions being somewhat less serious and the functional and psychological evolution being favorable, given the possibility to continue the rehabilitation treatment and the psychological counseling (which under Oradea's conditions may be prolonged as necessary if a family financial support is possible), the evolution might be good. 
To complete "THE COMPREHENSIVE INTEGRATED INPATIENT REHABILITATION" [1, 2] concept and to find a possible social support we lacked the possible help of a psychiatrist, a social assistant and occupational therapist in our rehabilitation team.

\section{Discussions}

The success of the rehabilitation act is a multifactorial problem. Motivation is a key ingredient, but not a guarantee of success given the complexity of traumatic injuries, chronic pain, neurological implications and mental health $[5,6]$. The efforts of the rehabilitation team have been able to overcome the psychological barriers and provide the basis for motivation for acceptance and participation in all rehabilitation efforts.

Traditional rehabilitation results are measured in independence from ADLs (activities of daily living) and IADLs (instrumental activities of daily living) [1, 2].

F Through our rehabilitation team and the rehabilitation effort we largely accomplished this sequence.

The transition to social and to family reintegration would be the full success of the rehabilitation act. It consists of vocational rehabilitation, fulfilling / satisfying family and social ties [1, 2, 3]. In our cases, when we speak about vocational rehabilitation, we speak about the need to choose a professional activity which might be completed under the circumstances of particular limited physical resources.

$F$ Because of the inability of the supervised and integrated rehabilitation process to continue, and due to the absence of the social worker and the occupational therapist, this sequence is out of control.

$F$ In addition, in our study cases, in the conditions of the traumatic event and its multiple psychological and functional consequences, their career choice may not fit. Who can help them?

There are still many points that the organization of the Romanian rehabilitation system needs to check and to solve.

\section{Conclusions:}

1. Managing pain, somatic dysfunction and posttraumatic stress in a polytrauma case is a complex process that requires a multidisciplinary team, including psychological approach and the possibility of long term rehabilitation and social interventions.

2. The rehabilitation feature of our cases consisted of multiple daytime rehabilitation sessions adapted to the daily resources and needs of the patients in the presence of a competent psychological support, conditions in which the hospitalization time had shortened.

\section{Bibliography}

1. Mohan D, Tiwari G, Khayesi M and Nafukho F M. Road traffic injury prevention: training manual, Unit 5: Delivering post-crash care, 80-88. World Health Organization 2006. ISBN 9241546751

2. Strasser D C, MD, Uomoto J M, PhD, Smith S J, PhD. The interdisciplinary team and polytrauma rehabilitation: prescription for partnership. Arch Phys Med Rehabil Vol 89, January 2008, 179-180.

3. Cifu D X, MD, Lew H L, MD, PhD. Handbook of Polytrauma Care and Rehabilitation. Cap. 11. Pain, 6668. Demos Medical Publishing 2014. E-book ISBN 9781936287550.

4. Wikipedia, Pain. https://en.wikipedia.org/wiki/Pain

5. Micaela Cornish Pop, Ph.D. The polytrauma experience: Clinical practice guideliness for cognitivecommunication rehabilitation. ASHA Convention November 18, 2006.

6. Tonkovic, Zarko Rasik, Tomislav Matejic. Stress and pain in emergency and trauma patients. PERIODICUM BIOLOGORUM UDC 57:61 VOL. 115, No 2, 135-138, 2013 